\title{
NOTES ON THE SPECIES OF THE PTERO- MEDUSAN GENUS TETRAPLATIA BUSCH, 1851
}

\author{
By Patricia M. Ralph ${ }^{1}$ \\ Zoology Department, Victoria University of Wellington, New Zealand
}

(Text-figs. I-3)

The affinities, structure and distribution of species of the genus Tetraplatia Busch, I85I have been discussed recently by Hand (1955), Beyer (I955) and Rees \& White (1957a), but ever since these curious bipyramidal medusae (without a typical medusan bell, marginal tentacles, or gastrovascular canals) were described by Busch they have provided much interest for the systematist. There is unanimous agreement that the clearly discernible nematocyst tracts, and the body wall of ectoderm and endoderm separated by a thick, welldefined mesogloea, place them within the Cnidaria as known at present. Tetraplatia is usually regarded as the only member of the hydrozoan order Pteromedusae, but Komai (I939) strongly advocates scyphozoan rather than hydrozoan affinities. The purpose of this paper, however, is not to discuss the broad relationships of the Pteromedusae, but to give further evidence that Tetraplatia chuni Carlgren, 1909 is a good species. (The structure and affinities of Tetraplatia will be discussed in another paper.)

Tetraplatia possesses a body divided into apical and oral regions by an encircling horizontal groove: there are four equidistant radially situated locomotory lappets arising from the groove, two ectodermal (?) statocysts on the undersurface (adoral) of each lappet; the sexes are separate, and there are four endodermal (?) gonads-each gonad has an apical pair of finger-like processes and an oral pair, and the two pairs, are joined by a narrow isthmus in the region of the horizontal groove.

Two species, T. volitans Busch I85I and T. chuni Carlgren 1909, are known and described. T. chuni lacks flying buttresses between the oral and apical regions of the body and has narrower locomotory lappets than $T$. volitans. Hand's (I955) material from off the Pacific Coast of North America (all of which he recognized as $T$. volitans) showed some specimens in which the buttresses were incomplete or fewer in number than the usual four, and others in which there were three instead of four lappets. Hand also found that, although in general there was little variation in the ratio of the width of the lappet and the space between the lappets (i.e. this space being as wide as the

${ }^{1}$ Nuffield Fellow, r958. 
lappet itself), in one or two specimens the space between the lappets was wider, being about twice the width of the lappet, and thus approached the condition described for $T$. chuni. Hand considered that the variation shown by his material demonstrated that $T$. chuni differs little from $T$. volitans and that it may be an aberrant form of it. Rees \& White (1957a, b) working with material from the South Pacific and the North and South Atlantic Oceans recognized $T$. chuni as distinct. They regarded the absence of buttresses in $T$. chuni and the ratio of $\mathrm{I}: \mathrm{I}$ of the length of the apical to oral body regions as a significant and reliable character readily separating $T$. chuni from $T$. volitans. All the material I have examined also demonstrates that $T$. chuni is a good species. There is also evidence that the juveniles of the two species can be as easily recognized as the adults although they have a similar oral to apical body length ratio of $\mathrm{I}: \mathrm{I}$.

\section{MATERIAL AND METHODS}

Dr F. S. Russell very kindly made available to me I9o specimens of Tetraplatia volitans taken by R. V. 'Sarsia' from the Continental Shelf at the approaches to the English Channel and the Bay of Biscay, and transverse and longitudinal serial sections made by $\mathrm{Mr}$ A. C. G. Best. The material was collected in the summer months of May to September in the years 1955 to 1957. Ring nets were used in vertical and oblique hauls. The greatest depth fished was approximately $1000 \mathrm{fm}$ but on an average the fishing depth was to about $450 \mathrm{fm}$. Hauls were made at various times of the day and night but, as collection of Tetraplatia was incidental to other tasks and closing nets were not used, correlation is not possible between abundance, diurnal migration and depth, etc. Fifty-seven Tetraplatia were taken in nine hauls in I955, II 5 from six hauls in 1956 and I8 from three hauls in 1957 . The great majority of the 190 specimens were lightly stained with borax carmine in order to determine sex more readily.

In addition, Dr W. J. Rees and Mr E. White of the British Museum (Natural History) very kindly allowed me to examine all their material of Tetraplatia from R.R.S. 'Discovery II' ( $T$. volitans and T. chuni); 'Carnegie VII'; H.M.S. 'Research' and F.R.V. 'Explorer' (T. volitans), as well as transverse sections of a single specimen of $T$. volitans from 'Discovery' collections.

The author wishes to thank Dr F. S. Russell, Director of the Plymouth Marine Laboratory, Dr W. J. Rees and Mr E. White of the British Museum (Natural History) for allowing her to examine material of Tetraplatia and for useful advice and encouragement, and to Miss Patricia G. Conway, Exeter, for assistance with measurements of specimens and graphs. 


\section{TETRAPLATIA VOLITANS BUSCH}

\section{Material collected by R.V. 'Sarsia'}

Of the total number of 190 specimens, I 73 were available for measurement, the others being damaged or too strongly contracted for accurate measurement. These range in size from $2 . \mathrm{I} \times \mathrm{I} \cdot 4 \mathrm{~mm}$ to $9.45 \times 2 . \mathrm{Imm}$, the width being measured at the level of the horizontal groove across the body. The greatest number, 90 in all, were between $4.0 \mathrm{~mm}$ and $6.0 \mathrm{~mm}$ long and $\mathrm{r} .0 \mathrm{~mm}$ and $2.5 \mathrm{~mm}$ wide. Approximately $80 \%$ of the specimens showed a dome-shaped apical tip (Fig. I, H). The length of the oral region plotted against that of the apical region shows clearly that the length of the oral region is, in specimens above $3.0 \mathrm{~mm}$ long, consistently twice that of the apical region and may be more, that is, up to 5:I (Fig. 2). Juveniles, however, usually have an oral to apical length ratio of $\mathrm{I}$ : I. The ratio for both the juveniles and the adults is similar to that obtained by Hand (1955) and Rees \& White (1957b).

Oral and apical gonad-lengths, plotted against total body length, show a similar ratio to that of the body regions; the oral lobes are usually the longer in animals above $5.0 \mathrm{~mm}$ long. A difference in the length of the oral and apical portions of the gonad has been noted previously. Dantan (I925, Pl. I, fig. I) figures the oral and apical gonad pairs as approximately equal in length. Beyer's (1955) 'Brategg' specimens showed the oral pairs longer than the apical, particularly in the larger specimens. Beyer thought that the gonad in these large specimens may have been partly spent. The present material, however, indicates that a difference in length of the apical and oral portions of the gonad is probably related to the difference in length of these major body regions and that growth of the gonad in length parallels the growth in length of these regions in the animal as a whole.

Males outnumber females by approximately $5: \mathrm{I}$ in the present collection. This ratio is based on Ioo animals taken over a three-year period in which collections were made by 'Sarsia', and in which the oral region of the gonad is over $0.5 \mathrm{~mm}$ in length. Specimens with gonads shorter than $0.5 \mathrm{~mm}$ in the oral region were frequently of indeterminate sex.

In the female gonad (Fig. I, D) the width to length ratio is usually $\mathrm{I}: 2$ but may be $\mathrm{I}: 3$, and in the male gonad (Fig. $\mathrm{I}, \mathrm{C}$ ) which is narrower and more elongated the ratio is frequently $I: 3$ and ranges to $I: 5$. Also, the range of width in the female gonad, $0.2 \mathrm{I}-\mathrm{I} \cdot 05 \mathrm{~mm}$, is greater than in the male, $0.07-$ $0.49 \mathrm{~mm}$. The two extremes in male gonad width, nevertheless, occur only once, and by far the greatest number have a gonad between 0.21 and $0.28 \mathrm{~mm}$ in width.

All sections demonstrate that $T$. volitans has a very narrow waist, from $0.50-0.75 \mathrm{~mm}$ in diameter in animals whose total length ranges from 4.5 to $6.5 \mathrm{~mm}$. The width of the waist was measured across the body (excluding the buttresses) in the region of the horizontal groove. One specimen sectioned 


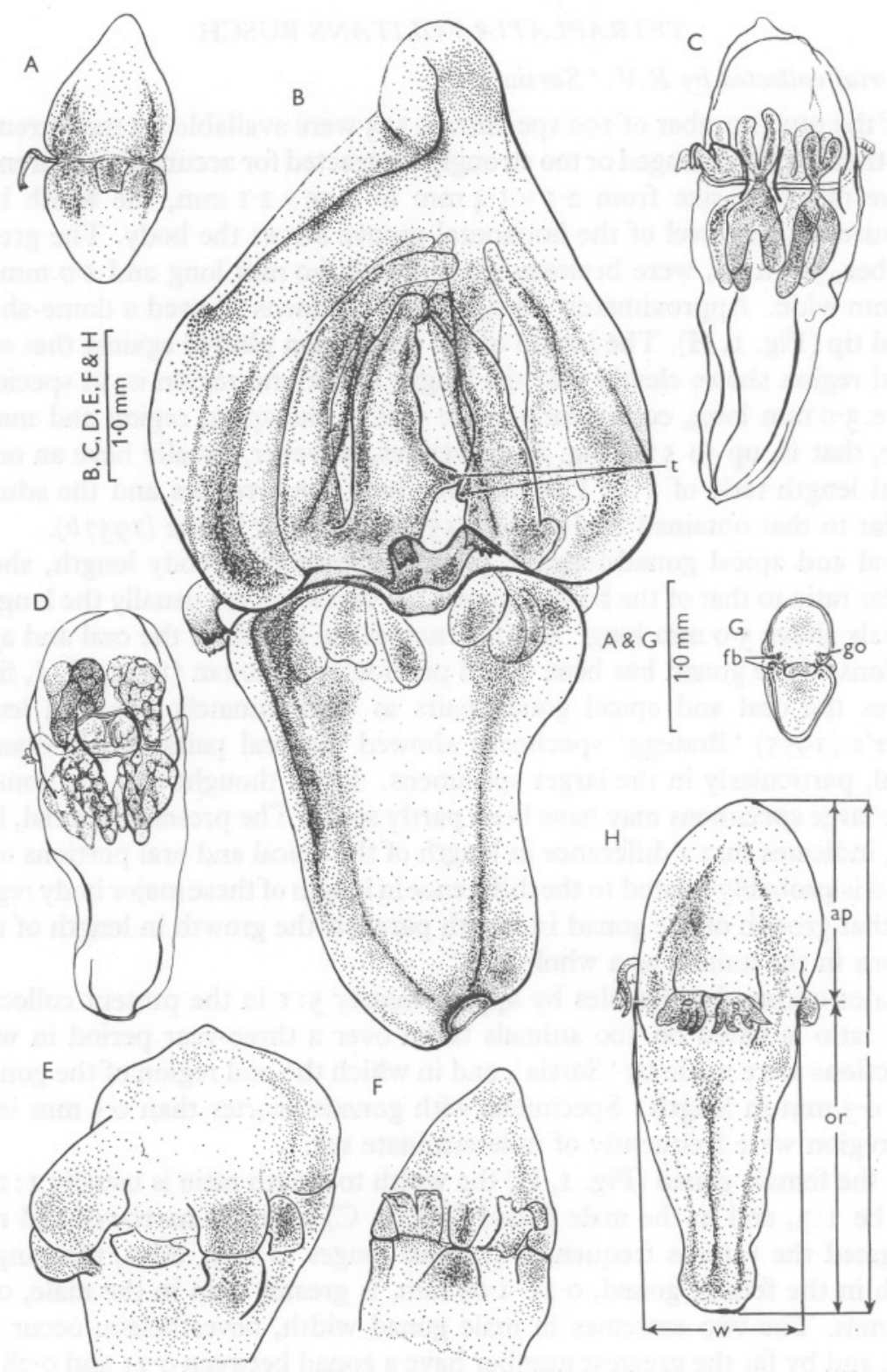

Fig. I. A and B, Tetraplatia chuni: A, juvenile (from N.W. Bouvet Is.); B, adult male (from north of the Antarctic ice edge); C-H, T. volitans: C, male, and D, female to show oral and apical paired finger-like portions of the gonad, E-G, specimens from R.R.S. 'Discovery II' collections, E, F, to show the aberrant flying buttresses. G, juvenile to show developing flying buttresses and incipient gonad; $\mathrm{H}$, diagram to show how specimens were measured.

Abbreviations: $a p$, measurement taken for length of the apical region; $f b$, flying buttress; go, gonad; $l$, total body length; or, measurement taken for length of oral region; $t$, small tubercle on male gonad of $T$. chuni; $w$, width of the body. 
longitudinally shows a distinct mesogloeal septum at the equator of two buttresses.

The position of the nematocyst tracts, the nematocysts, and the general external structure of the lappet are as described by Hand (1955). Twenty-five specimens measured for width of the lappet and distance between the lappets showed the usual ratio of $\mathrm{I}$ : I as known for the species. In no other specimen examined was there any significant departure from this ratio.

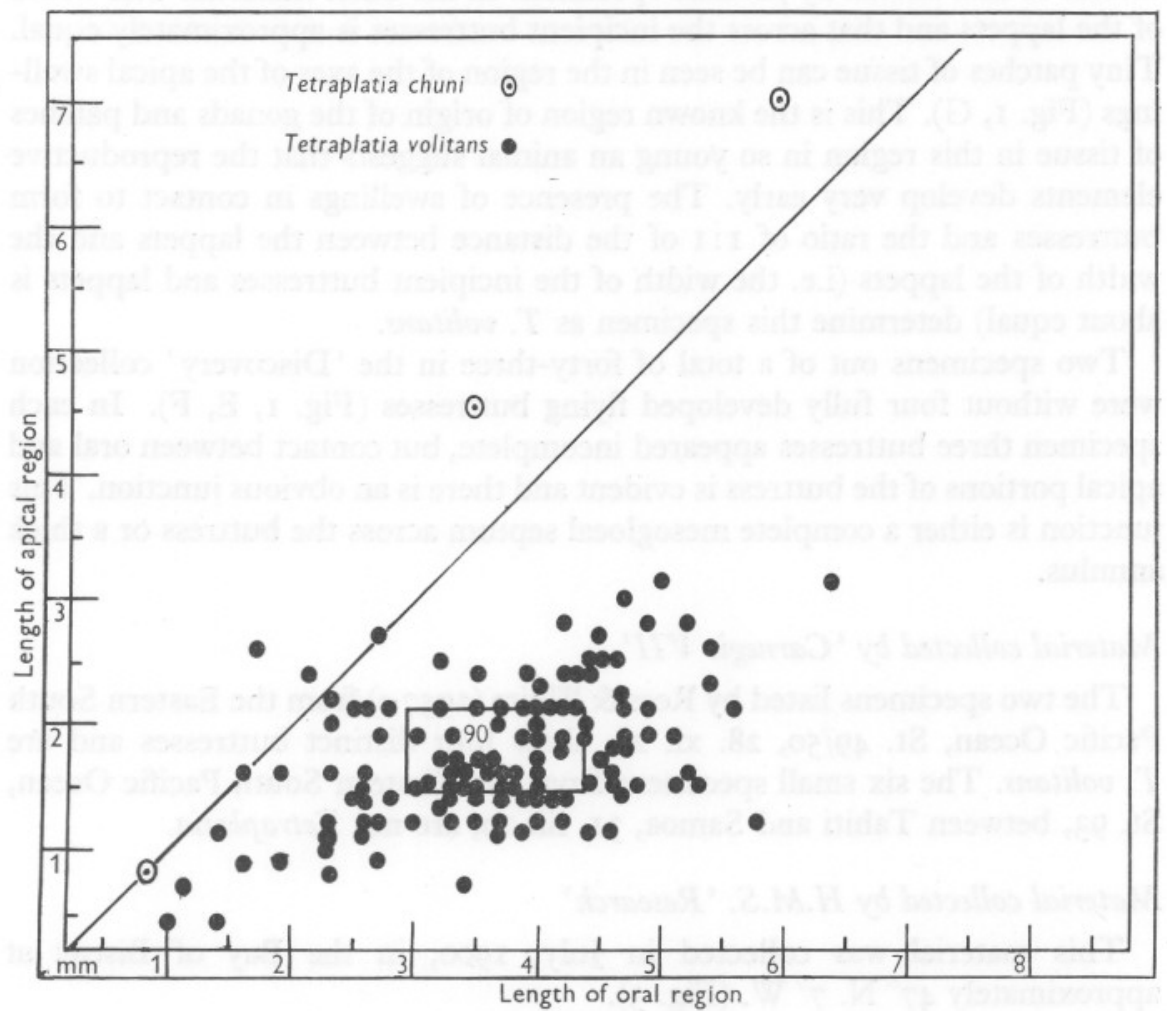

Fig. 2. Graph to show the ratio of the length of the oral region plotted against that of the apical region; line at angle of 45 degrees represents a I: I growth ratio of the oral/apical regions; rectangular area represents the ratio of the oral/apical growth length of the greatest number of specimens ( 90 in all).

\section{Material collected by R.R.S. 'Discovery II'}

Specimens examined of 'Discovery' material showed a similar oral: apical length ratio, gonad size and proportions, and ratio of males to females as did those of R.V. 'Sarsia', although gonad structure and sex were not so readily assessed as in the latter. One very young specimen $\mathrm{I} \cdot 0 \mathrm{~mm}$ in total length was noted (Fig. I, G). This has not been described before; it shows four equi- 
distant swellings at the posterior margin of the oral region and four corresponding swellings opposite them at the anterior margin of the apical region. There seems little doubt that these swellings are incipient buttresses, as there is contact between three of the pairs and in the remaining pair a linking strand of tissue. It is probable that all pairs become fused at this size and that the epithelial layer which is very thin and easily damaged has been torn away. The length ratio of the oral/apical body region is approximately as one is to one, similar to that shown by juvenile specimens in the other material. The width of the lappets and that across the incipient buttresses is approximately equal. Tiny patches of tissue can be seen in the region of the axes of the apical swellings (Fig. I, G). This is the known region of origin of the gonads and patches of tissue in this region in so young an animal suggests that the reproductive elements develop very early. The presence of swellings in contact to form buttresses and the ratio of $\mathrm{I}: \mathrm{I}$ of the distance between the lappets and the width of the lappets (i.e. the width of the incipient buttresses and lappets is about equal) determine this specimen as $T$. volitans.

Two specimens out of a total of forty-three in the 'Discovery' collection were without four fully developed flying buttresses (Fig. I, E, F). In each specimen three buttresses appeared incomplete, but contact between oral and apical portions of the buttress is evident and there is an obvious junction. This junction is either a complete mesogloeal septum across the buttress or a thick annulus.

\section{Material collected by ' Carnegie VII'}

The two specimens listed by Rees \& White (I957 a) from the Eastern South Pacific Ocean, St. 49/50, 28. xi. 28, show four distinct buttresses and are $T$. volitans. The six small specimens from the Western South Pacific Ocean, St. 93, between Tahiti and Samoa, 31. iii. 29, are not Tetraplatia.

\section{Material collected by H.M.S. 'Research'}

This material was collected in July, I900, in the Bay of Biscay at approximately $47^{\circ}$ N. $7^{\circ} \mathrm{W}$. (Fig. 3).

The two specimens from St. 320 and the single specimen from St. $35 b$ recorded by Rees \& White (1957a) have four fully developed buttresses and are similar to other material taken by R.V. 'Sarsia' of $T$. volitans in the Bay of Biscay. The specimen from St. $33 d$ is not Tetraplatia.

\section{TETRAPLATIA CHUNI CARLGREN, 1909}

Through the courtesy of Dr W. J. Rees and Mr E. White, of the British Museum (Natural History), I was able to examine material collected by R.R.S. 'Discovery II' from high latitudes in the South Atlantic and recognized by these authors as $T$. chuni. One specimen from north-west Bouvet Is. is a 
juvenile, $\mathrm{I} .75 \times 0.52 \mathrm{~mm}$, and the other from north of the Antarctic ice edge is a large mature male, $13.0 \times 4.0 \mathrm{~mm}$. The ratio of oral to apical body length in both these specimens is figured by Rees \& White (1957 b, fig. I), and in the present Fig. 2. In $T$. chuni, the oral region is about the same length as, or a little shorter than, the apical. In both specimens of $T$. chuni, the distance between lappets is approximately twice that of the width of the lappet-this is more apparent in the larger one.

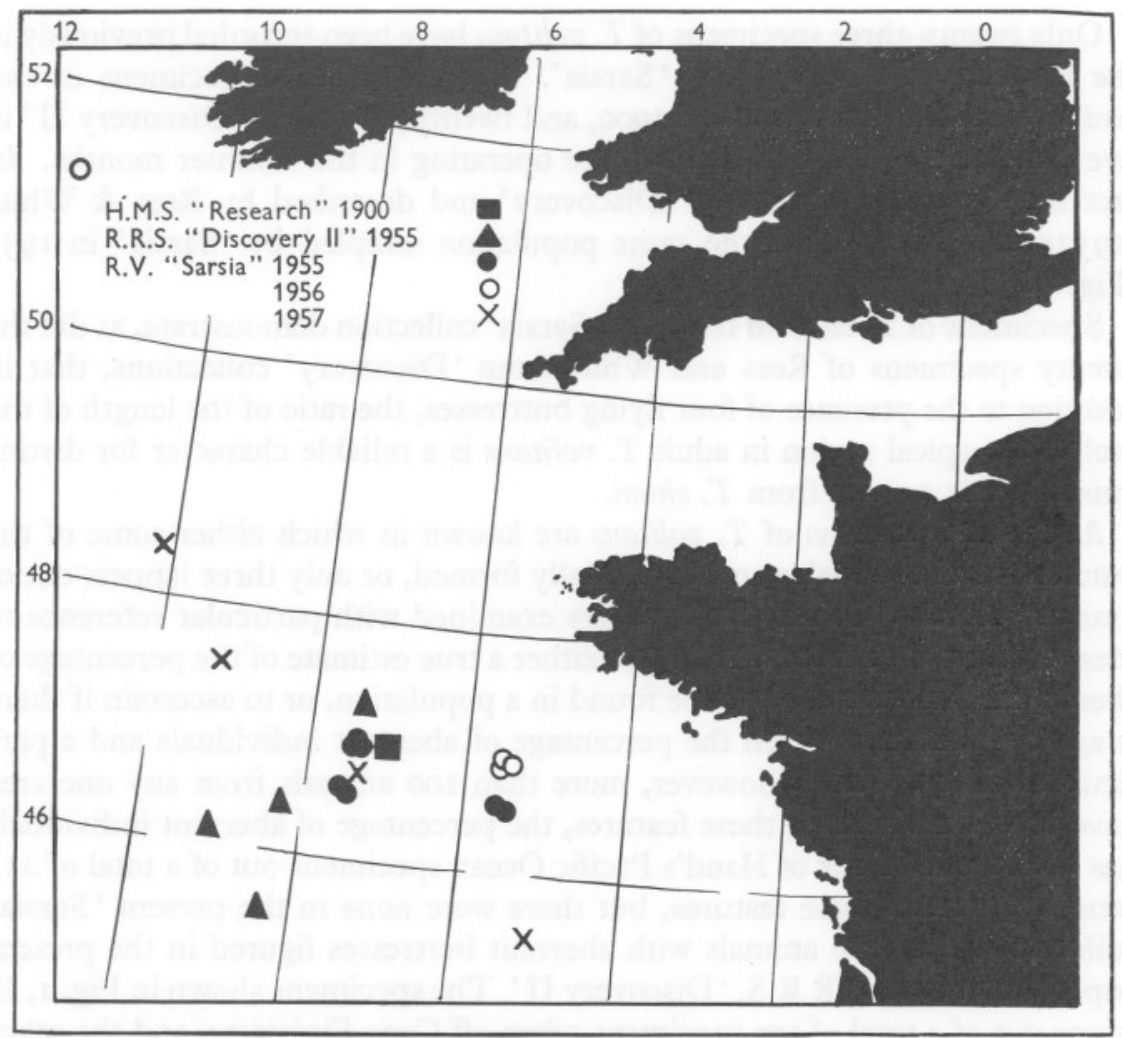

Fig. 3. Catches of $T$. volitans taken in the approaches to the English Channel.

In the large male the gonad is similar in shape (narrow and elongated) to that in $T$. volitans. There is however a distinct, small, outwardly directed tubercle on some of the apical gonad pairs (Fig. I, B). It has not yet been determined whether these tubercles have any connexion with the exterior by means of an indentation or 'pore' as is known for some material of $T$. volitans, or whether they are in any way involved in the shedding of the gonad products; their tips come very close to the external ectodermal epithelium.

The pairs of finger-like processes of the gonads in the apical region are 
greater in length $(4.5 \mathrm{~mm})$ than in the oral $(\mathrm{I} .5 \mathrm{~mm})$. As yet, there is insufficient material described to say whether this is always so, or whether it has any relationship to the proportions shown by the major body regions. Carlgren's (1909, Pl. XI, fig. 4) original specimen also had the male gonad in the apical region of greater length than the oral.

\section{REMARKS}

Only twenty-three specimens of $T$. volitans have been recorded previously in the area investigated by R.V. 'Sarsia'. They were three specimens in two hauls by H.M.S. 'Research' in I900, and twenty by R.R.S. 'Discovery II' in five hauls in 1955. All the ships were operating in the summer months. In fact the Tetraplatia taken by 'Discovery' and described by Rees \& White ( 1957 a) must be part of the same population sampled by 'Sarsia' in 1955 (Fig. 3).

Specimens of $T$. volitans from the 'Sarsia' collection demonstrate, as did the twenty specimens of Rees and White from 'Discovery' collections, that in addition to the presence of four flying buttresses, the ratio of the length of the oral to the apical region in adult $T$. volitans is a reliable character for distinguishing this species from $T$. chuni.

Aberrant specimens of $T$. volitans are known in which either some of the buttresses fail to develop, or are partially formed, or only three lappets out of four are present. So far, the numbers examined with particular reference to these features are inadequate to give either a true estimate of the percentage of aberrant individuals likely to be found in a population, or to ascertain if there is any correlation between the percentage of aberrant individuals and a particular locality. Where, however, more than roo animals from any one area have been examined for these features, the percentage of aberrant individuals has been small. Four of Hand's Pacific Ocean specimens out of a total of 2 I I were aberrant in these features, but there were none in the present 'Sarsia' collections. The two animals with aberrant buttresses figured in the present paper were taken by R.R.S. 'Discovery II'. The specimens shown in Fig. I, E, is one out of a total of ten specimens taken off Cape Finisterre, and the other (Fig. I, F), is one of two specimens from the South Atlantic Ocean. Measurements of Hand's (1955) 'more robust' specimens of $T$. volitans indicate that the width of the lappet may approximate to half the width of the distance between the lappets, i.e. approaching the ratio described for T. chuni. However, Hand did not find any in which the lappet was as narrow as that described by Carlgren for $T$. chuni and as shown by the specimens in the present 'Discovery' collection. All the 'Sarsia' specimens had the width of the lappet approximately equal to the length of the distance between the lappets, and were typical of $T$. volitans, irrespective of contraction due to preservation.

While only three specimens of $T$. chuni are known, they all show similar 
characters whether juvenile or adult. They are without flying buttresses, the oral and apical regions are almost equal in length, and they have narrow lappets, which are only half the width of the distance between the lappets. Juveniles of $T$. volitans, while possessing an oral and apical body region of equal length, have flying buttresses when approximately $\mathrm{I} \cdot 0 \mathrm{~mm}$ in length and lappets in which the width is approximately equal to the distance between them.

All in all, $T$. volitans and $T$. chuni as at present known can be readily recognized and their differing characters summarized as follows. First, and probably still of greatest significance for recognizing the two species, is the presence of four flying buttresses in juvenile and adult of $T$. volitans, and their absence in T. chuni. Secondly, the difference in ratio between the major body regions. In $T$. volitans the length of the oral region is much greater than in the apical - a ratio of at least $2: I$ and occasionally as much as 5:I in the adult. In $T$. chuni, on the other hand, oral and apical regions are approximately equal in length, but the apical part may be slightly longer. Thirdly, the width of the lappet in relation to the width of the distance between the lappets is different in the two species. In $T$. volitans the width of the lappet and the distance between the lappets is approximately the same, while in T. chuni the distance between the lappets is usually twice the width of the lappet. This relationship of distance between the lappets and the width of the lappet can be seen in both juveniles and adults.

Further, in $T$. volitans the gonad pairs of the oral region are longer than those of the apical part, while in T. chuni they are about half the length of the apical. In the former species the difference in length of the gonad pairs in the oral and apical regions seems to be correlated with the difference in length of the major body regions. As yet, there is insufficient evidence to say whether such a relationship is a constant character in T. chuni. Three other features are worthy of note: the very narrow waist of $T$. volitans and the much broader waist of T. chuni: the dome-shaped apical tip shown by the majority of preserved specimens of $T$. volitans compared with the bluntly pointed apical tip of $T$. chuni; and the greater size of $T$. chuni. Differences in apical shape are without doubt influenced by fixation and preservation, and therefore probably unreliable as a specific character, but the difference in apical shape in $T$. volitans and T. chuni may indicate a difference in behaviour and prove to be characteristic of the species. Similarly differences in size may be unreliable as a specific character, particularly in Tetraplatia of which T. chuni is as yet represented by only two adult specimens. Nevertheless, both these specimens are larger than the majority of adult $T$. volitans; one of them, from north of the Antarctic ice edge, is twice the size.

It seems that $T$. volitans with its four buttresses, larger lappets in relation to body width, and narrower waist, is more highly specialized than $T$. chuni. The sequence of events that led to this higher degree of specialization may have 
taken place as follows. First, there was the formation of flying buttresses by approximation of oral and apical outgrowths (of all three body-wall layers) and their fusion into a tubular, thick-walled strut between oral and apical regions of the body; the presence in the very tiny juvenile animal of the approximating outgrowths and the partial or complete septum of mesogloea in a few adult animals support this theory of the origin of the buttresses. At the moment, therefore, I cannot agree with Carlgren or Beyer (1955, p. II2) that the lack of buttresses in $T$. chuni is due to their degeneration from an originally buttressed condition. This conclusion is supported by the absence of buttresses in the juvenile T. chuni. Nor can I agree with Hand (1955, p. 337) that the sole function of the buttresses is to increase the digestive area, although this increase is incidental to the formation of buttresses. Their structure and shape make it reasonable to suppose that their primary function is now to afford some support for the body, even if, as indicated by the juvenile in the 'Discovery' collections, they originated as outgrowths from the gastric cavity. On the assumption that the buttresses give some support, a narrowing of the waist is possible and this would bring the lappets more closely into the groove between the buttresses, so that in effect the body becomes more streamlined. The neat fit of the lappets between the buttresses must also make for efficient swimming, as most, if not all of the locomotory beat would be effective, there being little or no lateral diversion of water. Increased growth of the oral body region may have arisen from increased swimming efficiency and the necessity for a highly mobile mouth as an efficient food catching mechanism. Tetraplatia chuni, on the other hand, lacking buttresses and having a long groove between narrow lappets, is probably a much less efficient swimmer than $T$. volitans. As yet, we may not have fished deeply enough to catch numbers of T. chuni.

\section{SUMMARY}

Collections of Tetraplatia made by R.V. 'Sarsia' from 1955 to 1957 are described and discussed in relation to specimens taken by R.R.S. 'Discovery II'; 'Carnegie VII'; H.M.S. 'Research' and F.R.V. 'Explorer'.

The results confirm that $T$. volitans Busch, I95I, and T. chuni Carlgren, 1909, are separate species which can be easily separated both in the juvenile and adult stages.

The difference in length of the gonad in the apical and oral regions in $T$. volitans is found to be correlated with the difference in length of these two major body regions. A similar gonad-length to body-length relationship can be seen in $T$. chuni, but insufficient material has been captured to ascertain whether this is a constant character in this species.

In general males occur in greater numbers than females in $T$. volitans. The known mature specimens of $T$. chuni are both males.

$T$. volitans is regarded as more highly specialized than $T$. chuni. 


\section{REFERENCES}

BEYER, F., I955. A new record of Tetraplatia (Pteromedusae). Nytt Mag. Zool. Oslo, Vol. 3, pp. 106-12.

Busch, W., I851. Beobachtungen über Anatomie und Entwicklung einiger wirbellosen Seetiere. I43 pp. Berlin.

Carlgren, O., 1909. Die Tetraplatien. Wiss. Ergebn. 'Valdivia', Bd. 19, Lief. 3, pp. $75-122$.

Dantan, J. L., 1925. Contribution à l'étude du Tetraplatia volitans. Ann. Inst. océanogr. Monaco, N.S. II, Fasc. 5, pp. 429-59.

HAND, C., 1955. A study of the structure, affinities and distribution of Tetraplatia volitans Busch (Coelenterata: Hydrozoa: Pteromedusae). Pacific Sci., Vol. 9, pp. 332-48.

KomaI, T., 1939. On the enigmatic Coelenterate Tetraplatia. Fap. F. Zool., Vol. 8, pp. $231-50$.

REES, W. J. \& WHITE, E., I957a. New observations on the aberrant medusa Tetraplatia volitans Busch. 'Discovery' Rep., Vol. 29, pp. 129-38.

REES, W. J. \& WHITE, E., I957b. Two new records of Tetraplatia chuni Carlgren from the South Atlantic. F. Fac. Sci. Hokkaido Univ., Ser. 6 (Zool.), Vol. I3, Nos. I-4, pp. IOI-4. 